\title{
Chronic Cough and Obesity
}

Laurent Guilleminault $\mathrm{MD}, \mathrm{PhD}^{1,2}$

1. Department of respiratory medicine, University Hospital Center of Toulouse, Toulouse, France

2. Center for Pathophysiology Toulouse Purpan, INSERM U1043, CNRS UMR 5282, Toulouse III University, Toulouse, France

Corresponding authors

L. Guilleminault

Pôle des voies respiratoires

CHU de Toulouse

24 chemin de Pouvourville

31059 Toulouse

Tel.: 0033567771850

Fax: 003356771472

guilleminault.1@chu-toulouse.fr 


\section{Abstract}

With respective prevalence of $13 \%$ and $9.6 \%$, obesity and chronic cough are two common conditions worldwide. The crucial role of obesity has been highlighted in the development and progression of many respiratory diseases. According to the results of epidemiological studies, obesity, particularly abdominal obesity, may also be associated with chronic cough (CC). CC seems to be more severe in obese patients compared to normal-weight subjects. The management of CC may differ slightly in obese patients compared to non-obese patients. Indeed, asthma and reflux diseases, which are considered key factors in the onset of CC, are characterised by more severe symptoms in obese patients. Asthma is associated with a resistance to usual treatments in obese patients but no data are available on the effect of inhaled therapies in obese subjects with cough variant asthma. Other emergent causes of CC have been reported in obese patients. Obstructive sleep apnoea and diabetes may also be involved in the development of $\mathrm{CC}$ and should be taken into account in obese patients with CC. The beneficial effect of weight loss on chronic cough has been suggested.

Key words: obesity, cough, asthma, GERD (gastroesophageal reflux disease). 


\section{Introduction:}

Obesity plays an important role in the development and progression of respiratory diseases. In asthma, obesity is considered a major comorbidity that exacerbates respiratory symptoms [1]. Many epidemiological studies have shown that obese patients have a higher risk of developing asthma than the general population demonstrating that obesity is involved in the development of asthma [2]. It is also well established that asthma associated with obesity is a phenotype with more difficult-to-treat asthma compared to non-obese subjects $[3,4]$. Obesity also plays an important role in chronic obstructive pulmonary disease (COPD) because it is associated with worse COPD-related outcomes and specific management of obesity reduces the risk of acute exacerbation of COPD [5]. In many lung diseases, weight loss is required to improve respiratory symptoms.

Chronic cough is a common entity in respiratory medicine. It is a complex disorder, the management of which has recently been defined in the guidelines of the American College of Chest Physicians [6]. All the steps covering the investigations and initial treatments are detailed in these guidelines. However, body mass index (BMI) measurement and assessment of obesity markers are not included in the evaluation of patients with chronic cough. As with other respiratory diseases, should obesity be taken into account in the management of chronic cough? Does obesity impact on the development of chronic cough? In this review, the link between chronic cough and obesity will be discussed as well as the clinical approach to an obese patient with chronic cough.

\section{Epidemiological data on chronic cough and obesity}

Over the last 40 years, obesity has tripled worldwide and is now considered a genuine public health problem [7]. In 2016, more than 1.9 billion people were overweight and more than 650 million were obese according to the World Health Organisation (WHO) [7]. Thirty-nine 
percent (39\%) of adults are overweight and 13\% are obese, worldwide [7]. Overweight and obesity are significantly associated with morbidity and mortality rates and a high BMI accounts for 4.0 million deaths globally [8]. Cardiovascular diseases and cancer are particularly involved in the mortality of obese patients $[9,10]$. Other diseases such as diabetes, hypertension, joint or digestive disorders are responsible for high morbidity rates in obese patients [11].

Chronic cough places high demands on health care systems but it is not classically associated with obesity. Chronic cough is a common disorder and its prevalence is estimated at $9.6 \%$ worldwide according to a meta-analysis [12]. In clinical practice, it is not surprising to observe the combination of two common diseases. However, is the association of chronic cough and obesity a coincidence or is there a link between these two disorders?

In their meta-analysis on the prevalence of chronic cough worldwide, Song et al. note that chronic cough is more frequent in Europe and America than in Asia and Africa [12]. The authors assume that comorbidities such as obesity might contribute to regional variability but this relationship was not examined in their meta-analysis. The guidelines on the management of cough in adults published in 2006 by Morice et al., specify that chronic cough is reported by 10 to $20 \%$ of adults and this disorder is more common in female and obese subjects [13]. Indeed, in a cross-sectional study involving 4003 patients, obesity (defined by a BMI $\geq 30$ $\mathrm{kg} / \mathrm{m}^{2}$ ) was associated with cough with an odds ratio (OR) of 1.66 [99\% CI (confidence interval) 1.19-2.32] in terms of univariate analysis. However, the difference was still not significant in the multivariate analysis, which suggests confounding factors [14]. More recently, Moralles-Estrella reported that patients with a higher BMI experience cough more often than other patients and the cough is more severe in obese patients [15].

BMI is a straightforward, easy-to-use marker but other obesity markers can also be used in clinical practice. Thus, waist-to-hip ratio seems to be more accurate in predicting 
cardiovascular risk [16]. The effect of various risk factors such as abdominal obesity on chronic cough was assessed in a large population-based cohort study performed in Denmark involving 14,669 adults. Abdominal obesity, defined as waist-hip ratio $>0.85$ for women and

$>0.90$ for men, was statistically more common in patients with chronic cough $(n=554)$ compared to healthy subjects (65\% vs. 53\%) [17]. In the total population, abdominal obesity was associated with chronic cough with an OR of 1.4 [1.2-1.7]. Interestingly, the authors ranked the risk factors according to the population attributable risk (PAR) calculated $[\mathrm{f}(\mathrm{OR}-$ $1)] /[1+\mathrm{f}(\mathrm{OR}-1)]$, where $\mathrm{f}$ is the frequency of the risk factor and $\mathrm{OR}$ is the odds ratio for chronic cough in the population. Using this formula, abdominal obesity was defined as the $3^{\text {rd }}$ risk factor in chronic cough, ranking higher than asthma, gastroesophageal reflux disease (GERD) or upper airway cough syndrome. When the results were stratified according to smoking status, abdominal obesity remains in the top ranking in former and current smokers according to PAR. However, in non-smokers, abdominal obesity is only the $7^{\text {th }}$ risk factor and the association with chronic cough is not significant (OR 1.3 [0.95-1.7]). Further studies are warranted to establish whether obesity plays a significant role in the development of chronic cough and whether comorbid conditions increase the prevalence of chronic cough in obese subjects.

$\underline{\text { Specificity of the clinical approach towards chronic cough in obese patients }}$

As explained above, obesity is a very common disease worldwide but its prevalence in patients with chronic cough has been inadequately studied. In the epidemiological study carried out in the UK in 2006, $20 \%$ of patients with chronic cough were obese [14]. This means that the proportion of obese patients with chronic cough is probably high in clinical practice. Should we manage chronic cough in obese patients differently from non-obese patients? The guidelines on chronic cough management may be applied in obese patients 
albeit with some specific features. The algorithm of chronic cough management in obese patients is outlined in Figure 1. Asthma, one of the main causes of chronic cough, presents differently in obese patients. In the cluster analysis conducted by Haldar et al., the authors found an obese asthma phenotype with non-eosinophilic inflammation [18]. The inflammatory profile of asthma may be different in obese patients compared to non-obese patients and the role of eosinophils in inflammation may be less crucial in obese asthmatics than non-obese asthmatics. However, sub-mucosal eosinophilic inflammation has also been found in asthma patients with obesity, which indicates that the association is probably more complex [19]. The obese-asthma phenotype is not a single entity and sub-phenotypes have been described depending on age, gender and asthma onset [20]. This probably involves various mechanisms of inflammation related to different endotypes. It is important to note that inhaled treatments appear to be less effective in obese patients with asthma. Indeed, there is evidence to show that inhaled corticosteroids and long-acting beta agonists are not as effective in asthma control in obese patients compared to non-obese patients [21, 22]. The real efficacy of inhaled therapies on cough has not been assessed in obese patients.

Cross-sectional epidemiological studies have demonstrated a higher prevalence of GERD in obese individuals compared to non-obese subjects. In a study involving 10,545 women, an increased risk of GERD was observed in obese patients with ORs of 2.92 (95\% CI 2.35-3.62) with a BMI of $30-34.9 \mathrm{~kg} / \mathrm{m}^{2}, 2.93(95 \%$ CI $2.24-3.85)$ with a $\mathrm{BMI} \geqslant 35 \mathrm{~kg} / \mathrm{m}^{2}$ compared to women with a BMI of $20-22.49 \mathrm{~kg} / \mathrm{m}^{2}$ [23]. Long-term complications of GERD such as erosive oesophagitis (EE), Barrett's oesophagus (BE), and oesophageal adenocarcinoma have been linked with obesity $[24,25]$. However, obesity does not seem to affect treatment outcomes with proton pump inhibitors (PPI). In patients with GERD, a high BMI was associated with more severe EE at baseline. However, during PPI treatment, BMI was not a significant independent predictor of heartburn resolution or EE healing [26]. When obese 
patients are treated with PPI therapy for reflux, no increased risk of BE or oesophageal carcinoma has been observed [27]. If a GERD-induced cough is suspected in clinical practice, PPIs should be tested [6]. PPIs are likely to have a similar effect on chronic cough in obese and non-obese patients. Robust evidence is needed to validate the use of PPIs in obese patients with GERD-induced cough.

A comprehensive history and physical examination are crucial in patients with chronic cough to rule out any life-threatening conditions and direct the investigation. In obese patients, clinicians should collect information relating to dietary habits, physical activity, eating patterns and the possibility of an eating disorder (binge eating disorder, night eating syndrome, bulimia), health consequences of obesity, patient expectations and motivation for change and previous treatments for obesity, according to the guidelines on the management of obese patients [28]. In addition to weight and height, waist and hip circumference should be measured especially during the follow-up. Indeed, waist and hip circumference is a better marker of adiposity especially visceral adiposity than BMI and it has been shown that waist circumference is significantly associated with hypertension, type 2 diabetes and OSAS [29]. Blood pressure should be routinely recorded in obese patients. Clinicians should be aware of obesity-related diseases such as diabetes, dyslipidaemia, cardiovascular, respiratory and joint diseases, and sleep disorders. As described below, some obesity-related diseases have an impact on chronic cough.

Minimal investigations in chronic cough should be carried out in order to determine the causes involved in cough. Fraction exhaled nitric oxide (FENO) is a helpful easy-to-use tool in patients with chronic cough because a high level of FENO is associated with cough variant asthma (CVA) and may predict the response to inhaled corticosteroids [30]. Obese individuals have a slight but significant increase in FENO compared to underweight individuals and obesity should be taken into account when FENO is performed in a context of chronic cough 


\section{$\underline{\text { Chronic cough and OSA }}$}

It is a well-established fact that obesity is a risk factor for the development and progression of obstructive sleep apnoeas (OSA) [32]. The prevalence of OSA in obese patients is nearly twice that of normal-weight adults [33]. An association between chronic cough and OSA was initially proposed in 2007 [34]. Birring et al. reported 4 patients presenting with an isolated chronic cough who were subsequently found to have obstructive sleep apnoea. Cough improved rapidly with nocturnal continuous positive airway pressure (CPAP) therapy. In a retrospective study carried out in 75 patients with chronic cough associated with normal lung function test and chest $\mathrm{X}$ ray, the proportion of patients with sleep disorder breathing was as high as $44 \%$ [35]. Amongst them, $42 \%$ had severe OSA defined by an index of hypopnea and apnoea (IHA) $\geq 30 / \mathrm{h}$. Interestingly, GERD was found in $52 \%$ of patients with sleep disorder breathing. Ninety-three percent of the patients who underwent procedures to optimise sleepdisrupted breathing witnessed an improvement in their cough. In a prospective study carried out in 53 patients referred for the evaluation of a sleep-breathing disorder and having OSA, chronic cough was observed in $33 \%$ of patients [36]. Compared to patients without chronic cough, those with chronic cough were mostly females and nocturnal heartburn, dysphagia, chest pain and rhinitis were more common. In another study, chronic cough was observed in $39.4 \%$ of patients with OSA and only $12.5 \%$ of patients without OSA suggesting chronic cough is particularly associated with OSA [37]. Mean BMI was statistically higher in patients with OSA highlighting the importance of obesity in the development of OSA. In the multivariate analysis, GERD was statistically associated with chronic cough in patients with OSA with an OR of 10.4 .

The mechanisms potentially responsible for the association of chronic cough with OSA have 
not been yet elucidated. As described previously, GERD is consistently found in studies that have focused on OSA and chronic cough. Therefore, GERD is put forward by many authors as the primary mechanism for the development of cough in OSA. Intra-thoracic and intraabdominal pressure changes during apnoea have been associated with the origin of GERD in OSA, but the mechanisms are probably more complex because GERD may interrupt sleep [38]. Upper and lower airway inflammation is observed in OSA and may also contribute to chronic cough [39, 40]. Trauma to the upper airways during each episode of apnoeahypopnoea could also contribute to the development of cough [41]. According to some authors, oedema and inflammation of the soft palate, uvula and supra-laryngeal soft tissues observed during OSA may predispose patients to increased sensitivity to coughing and especially to the "tickling" sensation often experienced by patients with chronic cough [42].

CPAP therapy is classically used in OSA to improve IHA and may be effective in the treatment of cough in OSA patients. In a prospective study, 28 patients with chronic cough were enrolled to assess the effect of CPAP therapy on cough [43]. In this study, the STOPBANG questionnaire was used to select the patients with a high probability of OSA. This questionnaire, based on symptoms and anatomical characteristics, predicts OSA with an intermediate risk if score is $\geq 3$. Twenty-two patients had a STOP-BANG score $\geqslant 3$, and among the 19 patients who accepted polysomnography and received CPAP treatment 7 had severe OSA. Leicester cough questionnaire (LCQ) score significantly improved after CPAP treatment. CPAP therapy seems to be of interest and may tackle cough hypersensitivity as suggested in a case report [44]. Indeed, cough reflex, measured by the citric acid test, improved after CPAP treatment and deteriorated after CPAP withdrawal. Moreover, in this case report, airway $\mathrm{pH}$ improves after CPAP therapy, which once again suggests the involvement of GERD in the association of chronic cough and OSA. Randomised controlled trials are needed in order to establish the real effect of CPAP treatment on chronic cough in 
OSA patients.

\section{$\underline{\text { Chronic cough and diabetes }}$}

Obesity is a recognised risk factor of type 2 diabetes mellitus [45]. In a study carried out in 33,939 men, obesity was associated with type 2 diabetes with an OR of 3.88 [95\% CI $1.94-$ 7.77] [46]. Recently, it has been suggested that type 2 diabetes may be a risk factor of chronic cough. Indeed, in a cross-sectional study, self-reported chronic cough/phlegm was more common in diabetic patients compared to the general population but only in patients 45-64 years old [47]. Indeed, in those patients, chronic cough/phlegm was reported by $27.6 \%$ of diabetic patients and $16.7 \%$ of the general population. No difference was observed between each group after 65 years of age. The prevalence of chronic cough increases in the general population after 65 years of age and the same prevalence was observed between both groups. Other causes of cough (GERD, COPD, Drugs, other respiratory diseases...) may appear with age and could explain why no difference was observed between diabetics and non-diabetics after 65 years of age. Further studies are needed to confirm the link between cough and diabetes.

Interestingly, it has been described that gliptin, a dipeptidyl peptidase- 4 inhibitor used in diabetes mellitus, is involved in drug-induced cough [48-50]. The prevalence of cough was $1.7 \%$ vs. $1.0 \%$ in the placebo arm of a randomised controlled study [48]. The mechanism seems to be similar to that observed with angiotensin converting-enzyme inhibitors [49].

\section{$\underline{\text { Weight loss and chronic cough }}$}

Given the fact that inhaled corticosteroids are less effective in obese patients with asthma, weight loss has been suggested as an option in obese patients with asthma [51]. The combination of dietary intervention and exercise has been shown to improve asthma control in 
obese patients [52]. Despite a lack of evidence, diet and exercise intervention may be considered in obese patients with chronic cough associated with asthma. A significant improvement in the ACQ (Asthma Control Questionnaire) score has also been observed with bariatric surgery in obese patients with asthma and this procedure may be interesting in obese asthmatics [53]. In obese patients with cough variant asthma, no data are available to support the beneficial effect of bariatric surgery on chronic cough.

Weight loss procedures are also linked to an improvement in GERD-related symptoms. In a cross-sectional survey performed on 44,997 patients between 2006 and 2009, weight loss was associated with fewer symptoms in a dose-dependent manner [54]. A structured weight loss program is needed to significantly reduce GERD symptoms. Indeed, in a prospective cohort study carried out on overweight/obese subjects (BMI 25-39.9 kg/m2), a structured weight loss program was assessed. This included dietary changes, increased physical activity and behavioural changes. A significant decrease in the overall prevalence of GERD (15 vs. 37\%) and the mean GERD symptom score (1.8 vs. 5.5) was observed in patients included in the treatment arm compared to patients in the control arm [55]. In patients with GERD-induced cough, the traditional dietary changes combined with weight reduction advice in the form of an Energy Prescription (EP) led to LCQ improvement compared to traditional dietary changes alone. Interestingly, a significant correlation between baseline high calorie and LCQ scores or fat intake and LCQ scores was observed. The role of diet or adipose tissue on chronic cough should be studied to enhance our knowledge of cough in obese patients.

\section{$\underline{\text { Conclusion }}$}

The link between obesity and chronic cough has been suggested in the literature. In the guidelines on chronic cough management, no difference has been highlighted between obese and normal-weight individuals. However, emergent causes of chronic cough such as OSA or 
diabetes should be taken into account in clinical practice. The beneficial effect of weight loss has been demonstrated in many obesity-related diseases. Weight-loss procedures may constitute part of the treatment of chronic cough in obese patients but further studies are warranted to support the use of these interventions in chronic cough. 


\section{References}

[1] C. Porsbjerg, A. Menzies - Gow, Co - morbidities in severe asthma: C linical impact and management, Respirology 22(4) (2017) 651-661.

[2] D.A. Beuther, E.R. Sutherland, Overweight, obesity, and incident asthma: a meta-analysis of prospective epidemiologic studies, American journal of respiratory and critical care medicine 175(7) (2007) 661-666.

[3] P. Saint - Pierre, A. Bourdin, P. Chanez, J.P. Daures, P. Godard, Are overweight asthmatics more difficult to control?, Allergy 61(1) (2006) 79-84.

[4] W.C. Moore, D.A. Meyers, S.E. Wenzel, W.G. Teague, H. Li, X. Li, R. D'Agostino Jr, M. Castro, D. Curran-Everett, A.M. Fitzpatrick, Identification of asthma phenotypes using cluster analysis in the Severe Asthma Research Program, American journal of respiratory and critical care medicine 181(4) (2010) 315-323.

[5] T. Goto, Y. Tsugawa, M.K. Faridi, C.A. Camargo Jr, K. Hasegawa, Reduced risk of acute exacerbation of COPD after bariatric surgery: a self-controlled case series study, Chest 153(3) (2018) 611-617.

[6] R.S. Irwin, C.L. French, A.B. Chang, K.W. Altman, T.M. Adams, E. Azoulay, A.F. Barker, S.S. Birring, F. Blackhall, D.C. Bolser, Classification of Cough as a Symptom in Adults and Management Algorithms: CHEST Guideline and Expert Panel Report, Chest 153(1) (2018) 196-209.

[7] Obesity and overweight [Internet]. World Health Organization. [cited 2018 Nov 7]. Available from: http://www.who.int/news-room/fact-sheets/detail/obesity-and-overweight. [8] G.O. Collaborators, Health effects of overweight and obesity in 195 countries over 25 years, New England Journal of Medicine 377(1) (2017) 13-27.

[9] P.S. Collaboration, Body-mass index and cause-specific mortality in 900000 adults: collaborative analyses of 57 prospective studies, The Lancet 373(9669) (2009) 1083-1096.

[10] A. Berrington de Gonzalez, P. Hartge, J.R. Cerhan, A.J. Flint, L. Hannan, R.J. MacInnis, S.C. Moore, G.S. Tobias, H. Anton-Culver, L.B. Freeman, Body-mass index and mortality among 1.46 million white adults, New England Journal of Medicine 363(23) (2010) 22112219.

[11] M. Abdelaal, C.W. le Roux, N.G. Docherty, Morbidity and mortality associated with obesity, Annals of translational medicine 5(7) (2017).

[12] W.-J. Song, Y.-S. Chang, S. Faruqi, J.-Y. Kim, M.-G. Kang, S. Kim, E.-J. Jo, M.-H. Kim, J. Plevkova, H.-W. Park, The global epidemiology of chronic cough in adults: a systematic review and meta-analysis, European Respiratory Journal (2015) ERJ-02187-2014.

[13] A. Morice, L. McGarvey, I. Pavord, Recommendations for the management of cough in adults, Thorax 61(suppl 1) (2006) i1-i24.

[14] A.C. Ford, D. Forman, P. Moayyedi, A.H. Morice, Cough in the community: a cross sectional survey and the relationship to gastrointestinal symptoms, Thorax 61(11) (2006) 975979.

[15] J.L. Morales-Estrella, F.D. Ciftci, W.E. Trick, K. Hinami, Physical symptoms screening for cardiopulmonary complications of obesity using audio computer-assisted self-interviews, Quality of Life Research 26(8) (2017) 2085-2092.

[16] S. Murray, Is waist-to-hip ratio a better marker of cardiovascular risk than body mass index?, Canadian Medical Association Journal 174(3) (2006) 308-308.

[17] Y. Çolak, B.G. Nordestgaard, L.C. Laursen, S. Afzal, P. Lange, M. Dahl, Risk factors for chronic cough among 14,669 individuals from the general population, Chest 152(3) (2017) 563-573.

[18] P. Haldar, I.D. Pavord, D.E. Shaw, M.A. Berry, M. Thomas, C.E. Brightling, A.J. Wardlaw, R.H. Green, Cluster analysis and clinical asthma phenotypes, American journal of respiratory and critical care medicine 178(3) (2008) 218-224. 
[19] D. Desai, C. Newby, F.A. Symon, P. Haldar, S. Shah, S. Gupta, M. Bafadhel, A. Singapuri, S. Siddiqui, J. Woods, Elevated sputum interleukin-5 and submucosal eosinophilia in obese individuals with severe asthma, American journal of respiratory and critical care medicine 188(6) (2013) 657-663.

[20] E.R. Sutherland, E. Goleva, T.S. King, E. Lehman, A.D. Stevens, L.P. Jackson, A.R. Stream, J.V. Fahy, D.Y. Leung, A.C.R. Network, Cluster analysis of obesity and asthma phenotypes, PLoS One 7(5) (2012) e36631.

[21] M. Peters-Golden, A. Swern, S. Bird, C. Hustad, E. Grant, J. Edelman, Influence of body mass index on the response to asthma controller agents, European Respiratory Journal 27(3) (2006) 495-503.

[22] L.-P. Boulet, E. Franssen, Influence of obesity on response to fluticasone with or without salmeterol in moderate asthma, Respiratory medicine 101(11) (2007) 2240-2247.

[23] A.K. Kasyap, S.K. Sah, S. Chaudhary, R. Shrestha, D. Shrestha, Association between Body Mass Index and Gastroesophageal Reflux Symptoms in Nepalese Adult Population-A Single Centered Hospital Based Study, JCMS Nepal 11(2) (2015) 23-26.

[24] H.B. El-Serag, D.Y. Graham, J.A. Satia, L. Rabeneck, Obesity is an independent risk factor for GERD symptoms and erosive esophagitis, The American journal of gastroenterology 100(6) (2005) 1243.

[25] P. Chang, F. Friedenberg, Obesity and GERD, Gastroenterology Clinics 43(1) (2014) 161-173.

[26] P. Sharma, N. Vakil, J.T. Monyak, D.G. Silberg, Obesity does not affect treatment outcomes with proton pump inhibitors, Journal of clinical gastroenterology 47(8) (2013) 672677.

[27] S. Erridge, O.M. Moussa, P. Ziprin, A. Darzi, S. Purkayastha, Risk of GERD-Related Disorders in Obese Patients on PPI Therapy: a Population Analysis, Obesity surgery (2018) 1-8.

[28] V. Yumuk, C. Tsigos, M. Fried, K. Schindler, L. Busetto, D. Micic, H. Toplak, O.M.T.F.o.t.E.A.f.t.S.o. Obesity, European guidelines for obesity management in adults, Obesity facts 8(6) (2015) 402-424.

[29] A.-L. Borel, S. Coumes, F. Reche, S. Ruckly, J.-L. Pepin, R. Tamisier, N. Wion, C. Arvieux, Waist, neck circumferences, waist-to-hip ratio: Which is the best cardiometabolic risk marker in women with severe obesity? The SOON cohort, PloS one 13(11) (2018) e0206617.

[30] W.-J. Song, H.J. Kim, J.-S. Shim, H.-K. Won, S.-Y. Kang, K.-H. Sohn, B.-K. Kim, E.-J. Jo, M.-H. Kim, S.-H. Kim, Diagnostic accuracy of fractional exhaled nitric oxide measurement in predicting cough-variant asthma and eosinophilic bronchitis in adults with chronic cough: a systematic review and meta-analysis, Journal of Allergy and Clinical Immunology 140(3) (2017) 701-709.

[31] A. Uppalapati, S. Gogineni, J.R. Espiritu, Association between Body Mass Index (BMI) and fraction of exhaled nitric oxide (FeNO) levels in the National Health and Nutrition Examination Survey (NHANES) 2007-2010, Obesity research \& clinical practice 10(6) (2016) 652-658.

[32] J. Durán, S. Esnaola, R. Rubio, Á. Iztueta, Obstructive sleep apnea-hypopnea and related clinical features in a population-based sample of subjects aged 30 to $70 \mathrm{yr}$, American journal of respiratory and critical care medicine 163(3) (2001) 685-689.

[33] A. Romero-Corral, S.M. Caples, F. Lopez-Jimenez, V.K. Somers, Interactions between obesity and obstructive sleep apnea: implications for treatment, Chest 137(3) (2010) 711-719. [34] S.S. Birring, A.J. Ing, K. Chan, G. Cossa, S. Matos, M.D. Morgan, I.D. Pavord, Obstructive sleep apnoea: a cause of chronic cough, Cough 3(1) (2007) 7.

[35] K.M. Sundar, S.E. Daly, M.J. Pearce, W.T. Alward, Chronic cough and obstructive sleep 
apnea in a community-based pulmonary practice, Cough 6(1) (2010) 2.

[36] K. Chan, A. Ing, L. Laks, G. Cossa, P. Rogers, S. Birring, Chronic cough in patients with sleep-disordered breathing, European Respiratory Journal 35(2) (2010) 368-372.

[37] T.-Y. Wang, Y.-L. Lo, W.-T. Liu, S.-M. Lin, T.-Y. Lin, C.-H. Kuo, F.-T. Chung, P.-C.

Chou, P.-J. Chang, Y.-L. Ni, Chronic cough and obstructive sleep apnoea in a sleep

laboratory-based pulmonary practice, Cough 9(1) (2013) 24.

[38] H.-k. Jung, R.S. Choung, N.J. Talley, Gastroesophageal reflux disease and sleep

disorders: evidence for a causal link and therapeutic implications, Journal of

neurogastroenterology and motility 16(1) (2010) 22.

[39] I. Rubinstein, Nasal inflammation in patients with obstructive sleep apnea, The Laryngoscope 105(2) (1995) 175-177.

[40] M. Alkhalil, E. Schulman, J. Getsy, Obstructive sleep apnea syndrome and asthma: what are the links?, Journal of Clinical Sleep Medicine 5(01) (2009) 71-78.

[41] J.H. Boyd, B.J. Petrof, Q. Hamid, R. Fraser, R.J. Kimoff, Upper airway muscle inflammation and denervation changes in obstructive sleep apnea, American journal of respiratory and critical care medicine 170(5) (2004) 541-546.

[42] K.M. Sundar, S.E. Daly, Chronic cough and OSA: an underappreciated relationship, Lung 192(1) (2014) 21-25.

[43] K.M. Sundar, S.E. Daly, A.M. Willis, A longitudinal study of CPAP therapy for patients with chronic cough and obstructive sleep apnoea, Cough 9(1) (2013) 19.

[44] S. Faruqi, A. Fahim, A.H. Morice, Chronic cough and obstructive sleep apnoea: refluxassociated cough hypersensitivity?, European Respiratory Journal 40(4) (2012) 1049-1050.

[45] D.P. Guh, W. Zhang, N. Bansback, Z. Amarsi, C.L. Birmingham, A.H. Anis, The

incidence of co-morbidities related to obesity and overweight: a systematic review and metaanalysis, BMC public health 9(1) (2009) 88.

[46] G. Twig, A. Afek, E. Derazne, D. Tzur, T. Cukierman-Yaffe, H.C. Gerstein, A. Tirosh, Diabetes risk among overweight and obese metabolically healthy young adults, Diabetes care (2014) DC_140869.

[47] F. De Santi, G. Zoppini, F. Locatelli, E. Finocchio, V. Cappa, M. Dauriz, G. Verlato, Type 2 diabetes is associated with an increased prevalence of respiratory symptoms as compared to the general population, BMC pulmonary medicine 17(1) (2017) 101.

[48] G. Schernthaner, A. Barnett, A. Emser, S. Patel, J. Troost, H.J. Woerle, M. von Eynatten, Safety and tolerability of linagliptin: a pooled analysis of data from randomized controlled trials in 3572 patients with type 2 diabetes mellitus, Diabetes, Obesity and Metabolism 14(5) (2012) 470-478.

[49] C. Lieurade, C. Mailhol, D. Brouquieres, M. Dupuis, R. Escamilla, A. Didier, A new cause of drug-induced cough: The dipeptidyl peptidase-IV inhibitors, Revue des maladies respiratoires 33(1) (2016) 78-80.

[50] J.N. Baraniuk, M.J. Jamieson, Rhinorrhea, cough and fatigue in patients taking sitagliptin, Allergy, Asthma \& Clinical Immunology 6(1) (2010) 8.

[51] M. Jensen, P. Gibson, C. Collins, J. Hilton, L. Wood, Diet - induced weight loss in obese children with asthma: a randomized controlled trial, Clinical \& Experimental Allergy 43(7) (2013) 775-784.

[52] P.D. Freitas, P.G. Ferreira, A.G. Silva, R. Stelmach, R.M. Carvalho-Pinto, F.L.

Fernandes, M.C. Mancini, M.N. Sato, M.A. Martins, C.R. Carvalho, The role of exercise in a weight-loss program on clinical control in obese adults with asthma. A randomized controlled trial, American journal of respiratory and critical care medicine 195(1) (2017) 32-42.

[53] A. van Huisstede, A. Rudolphus, M.C. Cabezas, L.U. Biter, G.-J. van de Geijn, C. Taube, P.S. Hiemstra, G.-J. Braunstahl, Effect of bariatric surgery on asthma control, lung function and bronchial and systemic inflammation in morbidly obese subjects with asthma, Thorax 
(2015) thoraxjnl-2014-206712.

[54] E. Ness-Jensen, A. Lindam, J. Lagergren, K. Hveem, Weight loss and reduction in gastroesophageal reflux. A prospective population-based cohort study: the HUNT study, The American journal of gastroenterology 108(3) (2013) 376.

[55] M. Singh, J. Lee, N. Gupta, S. Gaddam, B.K. Smith, S.B. Wani, D.K. Sullivan, A.

Rastogi, A. Bansal, J.E. Donnelly, Weight loss can lead to resolution of gastroesophageal reflux disease symptoms: a prospective intervention trial, Obesity 21(2) (2013) 284-290. 
Table 1: Management of chronic cough in obese patients. * Family history, dietary habits, frequency and type of physical exercise, eating pattern and possible presence of an eating disorder (binge eating disorder, night eating syndrome, bulimia), presence of depression and other mood disorders, drugs, endocrine abnormalities, psychosocial factors, chronic stress, smoking cessation, previous treatments for obesity. ** diabetes, hypertension, dyslipidaemia, cardiovascular diseases, respiratory and joint diseases, sleep disorders. BMI: body mass index, GERD: gastro-oesophageal reflux, CPAP: continuous positive airway pressure, CT: computed tomography, FENO: Fraction exhaled nitric oxide, HRCT: high-resolution CT, ICS: inhaled corticocorticosteroids, LABA: long acting beta agonist, LTRA: leukotriene antagonist, OSA: obstructive sleep apnoea, PPI: pump proton inhibitor.

Cough for at least 8 weeks and BMI $\geqslant 30 \mathrm{k} / \mathrm{m}^{2}$

a. Patient characteristics:

- $\quad$ Life-threatening conditions

- Query: cough characteristics, history of obesity, etc.*...

- Sleep apnoea questionnaire

- Physical examination including waist-to-hip ratio, blood pressure, signs of obesityrelated conditions $* *$

- Chest X-ray

b. Consider the 4 most common causes of chronic cough and treat if present

- GERD

If clinically suspected, test with PPI and diet lifestyle change

- Asthma

Spirometry with reversibility \pm bronchial challenge test

Allergy evaluation

Treatment: ICS \pm LABA, LTRA, trigger avoidance, weight loss

- Upper Airways Cough Syndrome

Sinus imaging, nasofibroscopy, allergy evaluation

Treatment: antihistamine and/or nasal corticosteroids

- Non-asthmatic Eosinophilic Bronchitis

Sputum eosinophilia, FENO, allergy evaluation Treatment: ICS, trigger avoidance

c. Consider 2 emergent causes in obese patients

- OSA

Sleep questionnaire and, if positive, nocturnal polygraphy

Treatment: CPAP treatment

- Diabetes

Blood glucose levels and, if abnormal, HbA1c

Treatment: specific diabetic treatments

d. Weight loss interventions if the patient is motivated

e. Further investigations if cough proves refractory:

- 24-hour oesophageal $\mathrm{pH} / \mathrm{Impedance}$ monitoring

- HRCT (high-resolution computed tomography), sinus CT scan

- Swallowing evaluation

- Bronchoscopy

- Environmental assessment 